COMMENT

\title{
Comment on Future trends in clinical genetic and genomic services by Borle et al.
}

Elisa J. F. Houwink (iD ${ }^{1 凶}$

(c) The Author(s), under exclusive licence to European Society of Human Genetics 2022

European Journal of Human Genetics (2022) 30:505-506; https://doi.org/10.1038/s41431-022-01056-3

Genetic information has caused many of us to reflect, and discuss whether the creation of this information should be handled by the regular health system instead of a commercial market system (such as direct to consumer testing). However, health care systems all over the world are increasingly working in a hybrid model, and use eHealth and blended care integrated in daily medical practice [1]. Of course, health is of importance for the individual and society at large, but with a growing need for personalized care and prevention, and for cross-disciplinary approaches. Borle et al. point out in this issue, the uncertainty of the future of genetic and genomic service provision and provide some insight into perspectives of Canadian genetic professionals on the topic [2]. This new evidence is of utmost importance since everybody knows by now that genetic information derived from DNA analysis can help address challenges in personalized medicine and prevention. But implementation of this knowledge is only in the early stages and needs further evaluation and strategic planning and decision making. Advances in DNA analysis technology, science and international collaborations have shown us indeed that genetic medicine is changing, complicating implementation steps. Certainly, DNA analysis is increasingly used in health care, but so far only in specialized settings and for a limited number of health problems and, thus, is still not capitalizing on its full potential [3].

For example, many common diseases can be familial in nature caused by rare genetic variants in specific genes, such as in BRCA1 or BRCA2 for breast cancer, or LDLR for familial hypercholesterolemia. Many of these gene-disease relationships have been welldescribed thanks to decades of clinical genetic testing, and lead to demonstrated prevention of disease, when relatives are preventively tested after a relative patient carrier has been identified. The technology to screen for such predefined actionable genetic variants exists, but is not currently used as preventive tool in the more general (patient) population [4]. Additionally, genetic variants influencing the efficacy or adverse events in drug prescription have been well described, and even included in pharmacological guidelines, but widespread use to guide personalized treatment strategies and prevent adverse events is limited [5]. Finally, polygenic risk scores (PRS) for many diseases have developed from outcomes of Genome-Wide Association studies (GWAS) [6]. By combining hundreds to thousands of common genetic variants, each explaining a (usually small) proportion of the disease, such PRS can now explain up to $20-50 \%$ of the occurrence of common diseases. These PRS can be combined with the rare familial genetic variants in preventive genetic testing, can be calibrated to populations or diverse subpopulations therein, and will grow in predictive ability as they become more commonly used both in primary and secondary care. Individualized genetic risk profiles (rare variants, pharmacogenetics and PRS) are increasingly affordable, at any point in time, and have a potential to be implemented at an unprecedented large scale in health care, both in clinics and for prevention. Yet, this is not fully exploited in medical practice because the logistic, technical, societal, ethical, legal, and educational requirements must be determined while public engagement and governmental vision is lacking. To bridge this gap, I hope international consortia can unite with (inter) national experts across all required disciplines and engage with the citizens, patients and other stakeholders.

Borle et al.'s paper showed some surprising results [2]. Although the study participants agreed about the most likely scenario universal usage of noninvasive prenatal screening, populationbased genome-wide sequencing of unaffected individuals was the least likely scenario while this could potentially help in prevention or early diagnostics of disease or prevention of pharmacological side effects through pharmacogenetic gene sequencing. Participants did indeed expect that the need for genetic health care services would increase by 2030 due to changes in clinical guidelines and increased use of genome-wide sequencing. Perhaps differences in expectations about the use of GWAS as a diagnostic tool instead of a screening tool could indeed be caused by uncertainties about clinical utility and sustainability and some of the medical and ethical issues arising from broad screening approaches. However, screening tools can also be seen as predictive tools in combination with other tools such as family history taking or other classic risk factors such as blood pressure taking, laboratory diagnostics such as cholesterol or glucose levels. The participants did agree with this point of view of potential impact of expanding eligibility of guidelines for genetic testing focusing on cancer. However, GWAS studies are applicable to monogenic and other more common diseases as well, so whether these results can be extrapolated to other diseases is unclear and needs further study.

Another comment to make is the fact Borle et al. point out the urgent need for health human resource planning for clinical genetic and genomic services to provide adequate access for all individuals in need and the search for alternative service delivery models [2]. This need is universal, however further studies are needed on perspectives of a diverse set of stakeholders on the role of genetics

${ }^{1}$ Department of Public Health and Primary Care (PHEG), Leiden University Medical Center, Leiden, The Netherlands. ${ }^{凶}$ email: e.j.f.houwink@lumc.nl

Received: 10 January 2022 Accepted: 18 January 2022

Published online: 8 February 2022 
in primary and secondary care, their view on responsibilities, information management through eHealth and decision support systems, and their educational needs. Understanding this, is a crucial step towards developing successful genetics education and communication that supports physicians' behaviour and clinical performance in daily practice [1]. This could potentially lead to decreasing the pressure on clinical genetic professionals with only necessary referrals from primary and secondary care nonclinical geneticists if needed according to guidelines. Borle et al. also pointed out on the increased use of eHealth and telemedicine, which indeed is happening due to COVID-19 pandemic [2]. Wang et al recently published a very interesting article on the "Utility of a virtual counselor (VICKY) to collect family histories among vulnerable patient populations: a randomized controlled trial" [7]. The study compared the efficacy of a virtual genetic counselor to the My Family Health Portrait tool. The study showed that many of the literacy-related barriers to using traditional digital tools can be overcome and could even be used when collecting health histories from vulnerable populations. Perhaps different forms of eHealth applications integrated in daily medical care can support clinical performance and increase the use of genetic knowledge in daily practice in the near future.

\section{REFERENCES}

1. Houwink EJF, Kasteleyn MJ, Alpay L, Pearce C, Butler-Henderson K, Meijer E, et al. SERIES: eHealth in primary care. Part 3: eHealth education in primary care. Eur J Gen Pract. 2020;26:108-18.

2. Borle K, Kopac N, Dragojlovic N, Rodriguez Llorian E, Friedman JM, GenCOUNSEL Study, et al. Where is genetic medicine headed? Exploring the perspectives of
Canadian genetic professionals on future trends using the Delphi method. Eur J Hum Genet. 2022. https://doi.org/10.1038/s41431-021-01017-2.

3. Khoury MJ. No shortcuts on the long road to evidence-based genomic medicine. JAMA. 2017;318:27-8.

4. Scheuner MT, Sieverding P, Shekelle PG. Delivery of genomic medicine for common chronic adult diseases: a systematic review. JAMA. 2008;299:1320-34.

5. Overkleeft R, Tommel J, Evers AWM, den Dunnen JT, Roos M, Hoefmans M-J, et al. Using personal genomic data within primary care: a bioinformatics approach to pharmacogenomics. Genes. 2020;11:1443 https://doi.org/10.3390/genes11121443.

6. Polygenic Risk Score Task Force of the International Common Disease Alliance. Responsible use of polygenic risk scores in the clinic: potential benefits, risks and gaps. Nat Med. 2021;27:1876-84.

7. Wang C, Paasche-Orlow MK, Bowen DJ, Cabral H, Winter MR, Norkunas Cunningham T, et al. Utility of a virtual counselor (VICKY) to collect family health histories among vulnerable patient populations: a randomized controlled trial. Patient Educ Couns. 2021;104:979-88.

\section{COMPETING INTERESTS}

The author declares no competing interests.

\section{ADDITIONAL INFORMATION}

Correspondence and requests for materials should be addressed to Elisa J. F. Houwink.

Reprints and permission information is available at http://www.nature.com/ reprints

Publisher's note Springer Nature remains neutral with regard to jurisdictional claims in published maps and institutional affiliations. 